\title{
Seroprevalence of Toxoplasma gondii infection in blood donors in mainland China: a systematic review and meta-analysis
}

\author{
Taiwu Wang ${ }^{1, \dagger}$, Yifang Han $^{1, \dagger}$, Zuanqin Pan ${ }^{2}$, Hengzhong Wang ${ }^{2}$, Meng Yuan ${ }^{3}$, \\ and Hong $\operatorname{Lin}^{4, *}$ \\ 1 Research Institute for Medicine of Nanjing Command, 293 Zhongshan Eastern Road, Nanjing 210002, PR China \\ 2 Gaoyou Hospital Affiliated to Soochow University (Gaoyou People's Hospital), Gaoyou 225600, PR China \\ 3 Faculty of Preventive Medicine, The Fourth Military Medical University, 169 Changle West Road, Xi'an 710032, PR China \\ 4 Jiangsu Province Blood Center, 179 Longpan Road, Nanjing 210042, Jiangsu, PR China
}

Received 30 March 2018, Accepted 23 June 2018, Published online 23 July 2018

\begin{abstract}
Toxoplasma gondii transmitted from blood donors to receiving patients has become a concern as numerous articles about the epidemiology of $T$. gondii infection in blood donors from different provinces have been published in China. This study aimed to evaluate the seroprevalence of $T$. gondii infection in Chinese blood donors using a meta-analysis. A total of 40 eligible studies, published from 1986 to 2017 and covering 18 provinces and municipalities were included. Among a total of 49,784 Chinese blood donors, the overall IgG seroprevalence of T. gondii infection was $6.26 \%$ (95\% CI: $4.62 \%-8.13 \%)$. The highest prevalence was in the Northeast of China and the lowest in Central China. The infection rate increased slowly over the years, but not significantly. A statistically significant correlation was found between the seroprevalence of $T$. gondii infection and the detection method and educational level $(p<0.01)$. There was no relationship between age, gender, occupation and blood type and seroprevalence of T. gondii $(p>0.05)$. The prevalence of antibodies to T. gondii in Chinese blood donors was lower than in other countries, but the risk of transfusion-transmitted toxoplasmosis still exits. More concise methods are still needed to evaluate the possibility of transfusion-transmitted toxoplasmosis from blood donors.
\end{abstract}

Key words: Toxoplasma gondii, Blood donors, China, Systematic review, Meta-analysis.

\begin{abstract}
Résumé - Séroprévalence de l'infection par Toxoplasma gondii chez les donneurs de sang en Chine continentale: examen systématique et méta-analyse. La transmission de Toxoplasma gondii des donneurs de sang aux patients receveurs est devenue un sujet de préoccupation car de nombreux articles sur l'épidémiologie de l'infection à $T$. gondii chez des donneurs de sang de différentes provinces ont été publiés en Chine. Cette étude visait à évaluer la séroprévalence de l'infection par $T$. gondii chez des donneurs de sang chinois à l'aide d'une méta-analyse. Un total de 40 études admissibles, publiées de 1986 à 2017 et couvrant 18 provinces et municipalités, ont été incluses. Sur un total de 49784 donneurs de sang chinois, la séroprévalence globale des IgG de l'infection à $T$. gondii était de 6,26\% (IC à $95 \%: 4,62 \%-8,13 \%$ ). La prévalence la plus élevée était au nord-est de la Chine et la plus faible en Chine centrale. Le taux d'infection a augmenté lentement au cours des années, mais pas de manière significative. Une corrélation statistiquement significative a été trouvée entre la séroprévalence de l'infection par T. gondii et la méthode de détection et le niveau d'éducation $(p<0,01)$. Il n'y avait pas de relation entre l'âge, le sexe, la profession, le type sanguin et la séroprévalence de T. gondii $(p>0,05)$. La prévalence des anticorps contre $T$. gondii chez les donneurs de sang chinois est plus faible que dans les autres pays, mais le risque de toxoplasmose transmise par transfusion existe toujours. Des méthodes plus concises sont encore nécessaires pour évaluer la possibilité de toxoplasmose transmise par transfusion chez les donneurs de sang.
\end{abstract}

\footnotetext{
*Corresponding author: linhong712003@sina.com

$\dagger$ These authors contributed equally to this work.
} 


\section{Introduction}

Toxoplasmosis, a worldwide disease in humans and most warm-blooded animals, is caused by the opportunistic protozoan Toxoplasma gondii. One-third of the world's population is estimated to be infected by this parasite [25]. T. gondii infects humans mainly through oral, blood and congenital transmission [34]. T. gondii was discovered in 1908 [51] and first in 1955 in China, and the corresponding work was published in 1957 [51]. The first human case of toxoplasmosis in China was reported in 1964 [44]. Over the last few decades, epidemiological surveys have been conducted to monitor the prevalence of $T$. gondii in China. The prevalence was $5.17 \%$ $(0.33 \% \sim 11.79 \%)$ in the first national investigation between 1988 and 1992 [52], and then rose to about 7.9\% between 2001 and 2004 [56], and 12.3\% between 2006 and 2008 [43]. As an opportunistic pathogen, T. gondii rarely causes serious symptoms in healthy humans. However, the prevalence of T. gondii infection is rising and the number of clinical cases in immunocompromised patients [57] is increasing, such as transplant recipients, HIV-positive individuals, and cancer patients, as well as patients with congenital toxoplasmosis and psychosis. More attention should be given to toxoplasmosis as a serious public health problem.

It has been confirmed that $T$. gondii is a transfusion-transmissible pathogen [29]. In a meta-analysis, the prevalence of T. gondii in blood donors was estimated to be $33 \%$ worldwide [8]. In China, researchers paid more attention to $T$. gondii during the 1980s-1990s than at present. T. gondii screening in blood donors was piloted in many provinces. Based on these screening data, $T$. gondii infection deferral was added to Blood Donor Healthy Check Guidelines in 2001 [22]. The rule stipulates that toxoplasmosis recovered blood donors should be deferred for six months. Although T. gondii infection rates in Chinese blood donors have increased, rates are still relatively low compared with other countries [8]. In recent years, knowledge of $T$. gondii was low, and raw meat consumption and exposure to domestic pets has increased. Most blood donors are surprised by questions on $T$. gondii in questionnaires, and ask what toxoplasmosis is. So far, a comprehensive study on the prevalence of $T$. gondii has not been performed. Therefore, we conducted a national systematic review and meta-analysis to assess the prevalence of antibodies to T. gondii in Chinese blood donors.

\section{Methods}

This study is based on the preferred reporting items for systematic reviews and meta-analyses (PRISMA) checklist [23], used to search for and select studies, and to assess quality and extracted data. This was done by two researchers, independent of each other, to avoid bias.

\section{Search strategy}

Firstly, an inductive electronic search using keywords for all potential articles was performed; secondly, a deductive approach was used by searching for and retrieving articles from reference sections of identified publications as well as review articles related to blood donors or donations in China. We searched for epidemiological studies that were conducted before December 2017 in five English-language databases, including PubMed, Springer Link, Science Direct, Web of Science, and Wiley Online Library, and three Chinese databases: Wanfang, China National Knowledge Infrastructure (CNKI), and VIP (WeiPu). The keywords used to search the databases were Toxoplasma, Toxoplasma gondii, T. gondii, Toxoplasmosis, blood donation, blood donors, transfusion, Chinese and China. To maximize outputs, each keyword was searched individually or in combination. Result agreement and discrepancies between results were examined by a third researcher.

\section{Inclusion and exclusion criteria}

Selected manuscripts needed to fulfill the following inclusion criteria: (i) cross-sectional study; (ii) locations within mainland China; (iii) targeted objectives were blood donors; (iv) serological diagnostic methods of IgG were used; (v) exact total and positive numbers were provided; and (vi) a sample size greater than 100. Studies were excluded if they did not fulfill all these criteria.

\section{Data extraction}

The desired data were recorded using a data extraction form which included title, year of publication, province, sample size, number of seropositive cases, and diagnostic methods by two reviewers (Wang and Lin) using the inclusion criteria. Data on risk factors such as gender, age, education level, occupation and blood groups were also extracted. Discrepancies were resolved by discussion between the two reviewers and by seeking the opinion of the third author (Pan), if necessary.

\section{Meta-analysis}

To avoid the confidence interval (CI) being out of the $0-1$ range, and to prevent a study from having a large weighting when the proportion becomes too small or too large [2], we calculated seroprevalence estimates with variance stabilizing double arcsine transformation [2]. In addition, if the prevalence obtained from studies was not normally distributed, the prevalence needed to be transformed.

Point estimates and their 95\% confidence intervals (CIs) for the prevalence rate of antibodies to toxoplasmosis were calculated for each study. The random effects model was adopted for overall and subgroup analysis if obvious heterogeneity existed, otherwise the fixed effects model was used. Furthermore, both models were adopted to test the difference of the two models for sensitivity analysis. Statistical heterogeneity was evaluated by the Cochran Chi-squared test (with $p<0.10$ indicating statistically significant heterogeneity) and the statistic $I^{2}$ [6] (heterogeneity with $I^{2}$ of $0 \%-40 \%$ was considered not important, while $I^{2}$ of 30 to $60 \%$ was moderate heterogeneity, $I^{2}$ of $50 \%-90 \%$ was substantial heterogeneity, and $I^{2}$ of $75 \%-100 \%$ was considerable heterogeneity). A forest 


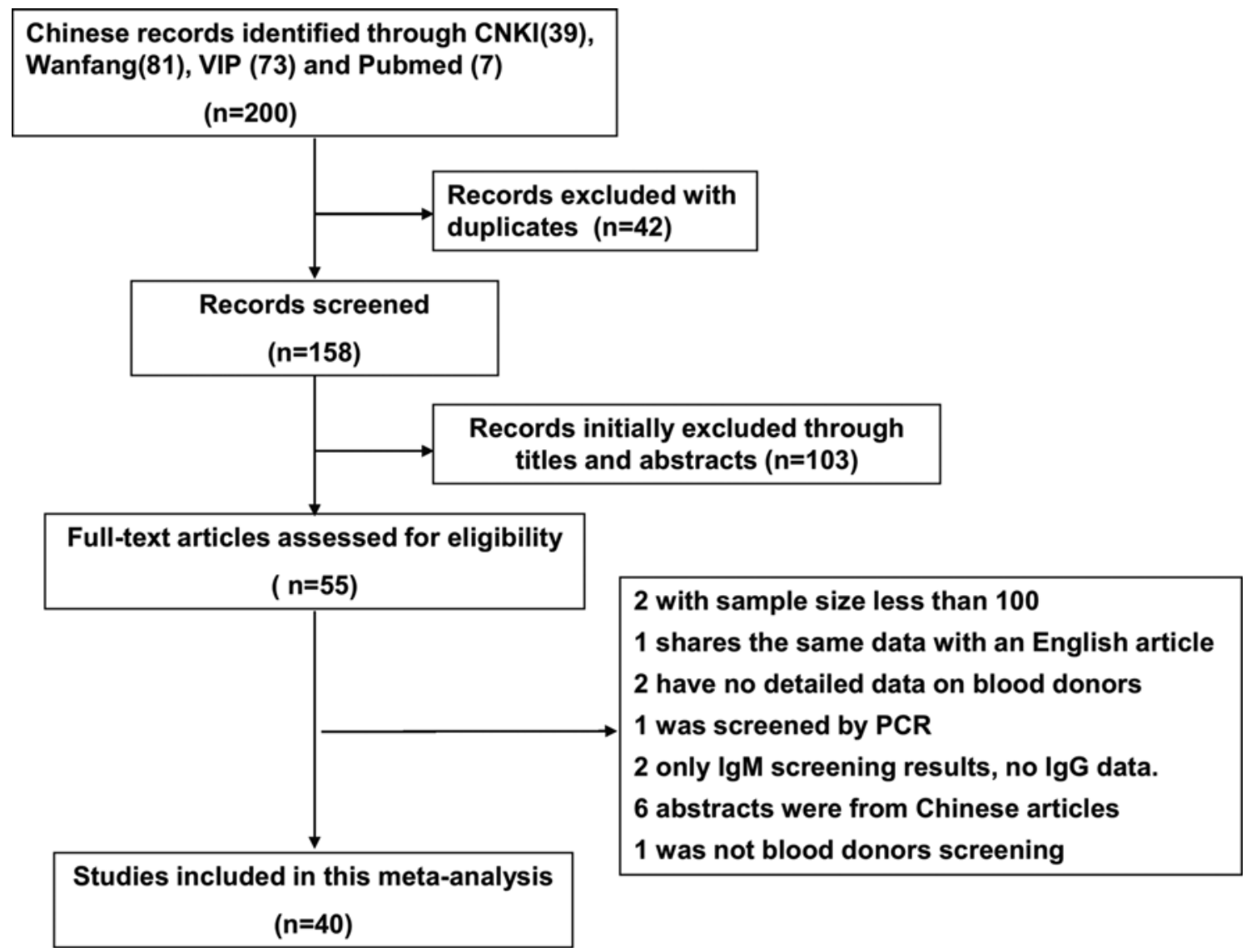

Fig. 1. Flowchart describing the study design process.

plot was used to provide a comprehensive overview of the included studies according to research year. Potential sources of heterogeneity were investigated further by arranging groups of studies according to potentially relevant characteristics. In this study, subgroup analysis was stratified by detection methods, regions (Northwest, Southwest, Northeast, South China, Central China, East China and North China), age, gender, occupation, blood group, and education background. Furthermore, meta-regression was used to investigate any significant difference between/among subgroups and the value of IgG seroprevalence. The publication bias was examined by funnel plots. In addition, the statistical significance was assessed by Egger's regression asymmetry test. For meta-analysis, we assumed that the included studies were a random sample from each study population. All analyses were carried out with $\mathrm{R}$ software version 3.4.1 (with the package "meta" [26] (version 4.8-4) for meta-analysis).

\section{Results}

\section{Characteristics of the eligible studies}

Through our systematic review, a total 200 articles were found following the initial database search (Fig. 1). Table 1 shows the characteristics of the 40 studies [1, 3, 4, 7, 9-18, $21,27,28,30-33,35,37-42,45,47-49,53-55,58-62]$ ultimately eligible for inclusion, which covered 18 provinces. The years in which the studies were performed and published ranged from 1985 to 2016 and from 1986 to 2017, respectively. The total number of blood donors was 49,784, with a range of 110-5068 per study, with one of two tests including EnzymeLinked Immunosorbent Assay (ELISA, $n=31$ ) [1, 4, 7, 10, $12,13,15-18,21,27,28,30-32,35,37-40,42,45,47,49$, 53-55, 58, 60, 62], and the Indirect Hemagglutination Test (IHA, $n=9)[3,9,11,14,33,41,48,59,61]$ (Table1).

\section{Baseline characteristics of blood donors in included studies based on geographic regions}

A total of 3578 blood samples were found to have $T$. gondii IgG antibodies and the overall prevalence in blood donors was $6.26 \%$ (95\% CI: $4.62 \% ; 8.13 \%)$. The forest plot diagram of the current meta-analysis is presented in Fig. 2.

Geographic analysis showed that the highest IgG prevalence of $T$. gondii infection was in Northeast blood donors (21.21\%, 95\% CI: $16.48 \%-26.36 \%)$ and the lowest in Central China (4.24\%, 95\% CI: 2.25\%-6.82\%) (Table 2). The prevalence rates of $T$. gondii in blood donors among different provinces are shown in Figure 3. The highest and lowest prevalence of $T$. gondii were found in Sichuan $(37.76 \%$, $95 \%$ CI: $32.66 \%-42.99 \%)$ and Hunan $(1.27 \%$, $95 \%$ CI: $0.68 \%-2.02 \%$ ), respectively. 
Table 1. Baseline characteristics of included studies based on geographical regions in China.

\begin{tabular}{|c|c|c|c|c|c|c|c|}
\hline Region & Province & Author [reference] & Method & Publication year & Population & Number of IgG-positive sera & Prevalence $(\%)$ \\
\hline Northwest & Shanxi & Ai et al. [16] & ELISA & 2007 & 368 & 30 & 8.15 \\
\hline Northwest & Gansu & Wang et al. [17] & ELISA & 1998 & 1480 & 150 & 10.14 \\
\hline Northwest & Xinjiang & Sun et al. [18] & IHA & 1991 & 328 & 24 & 7.32 \\
\hline Southwest & Guizhou & Chen et al. [19] & ELISA & 1999 & 500 & 32 & 6.40 \\
\hline Southwest & Guizhou & $\mathrm{Hu}$ et al. $[20]$ & IHA & 1991 & 200 & 1 & 0.50 \\
\hline Southwest & Sichuan & $\mathrm{Wu}$ et al. [21] & ELISA & 1989 & 339 & 128 & 37.76 \\
\hline Southwest & Yunnan & Zhu et al. [22] & ELISA & 2007 & 5068 & 1006 & 19.85 \\
\hline Southwest & Chongqing & $\mathrm{Xu}$ et al. [23] & ELISA & 2017 & 1001 & 85 & 8.49 \\
\hline Northeast & Heilongjiang & Wang et al. [24] & ELISA & 2002 & 264 & 56 & 21.21 \\
\hline North China & Hebei & Song et al. [25] & ELISA & 2009 & 792 & 38 & 4.80 \\
\hline North China & Hebei & Song et al. [26] & ELISA & 2012 & 1612 & 189 & 11.72 \\
\hline North China & Hebei & Wang et al. [27] & ELISA & 2014 & 832 & 35 & 4.21 \\
\hline North China & Hebei & Yang et al. [28] & ELISA & 2012 & 1056 & 51 & 4.83 \\
\hline North China & Hebei & Xin et al. [29] & ELISA & 2013 & 864 & 44 & 5.09 \\
\hline North China & Hebei & Wu et al. [30] & ELISA & 2017 & 1630 & 126 & 7.73 \\
\hline North China & Hebei & Shen et al. [31] & ELISA & 2017 & 1165 & 83 & 7.12 \\
\hline Central China & Henan & Yang et al. [32] & IHA & 1995 & 469 & 20 & 4.26 \\
\hline Central China & Henan & Luo et al. [33] & ELISA & 2003 & 960 & 50 & 5.21 \\
\hline Central China & Henan & Sun et al. [34] & ELISA & 2015 & 3200 & 98 & 3.06 \\
\hline Central China & Hubei & $\mathrm{Gu}$ et al. [35] & IHA & 1989 & 2063 & 32 & 1.55 \\
\hline Central China & Hubei & Kuang et al. [36] & ELISA & 2002 & 256 & 14 & 5.47 \\
\hline Central China & Hubei & Li et al. [37] & ELISA & 2003 & 584 & 79 & 13.53 \\
\hline Central China & Hunan & Tong et al. [38] & ELISA & 1994 & 1105 & 14 & 1.27 \\
\hline East China & Shandong & Feng et al. [39] & ELISA & 1998 & 2025 & 259 & 12.79 \\
\hline East China & Jiangsu & Zhu et al. [40] & IHA & 1987 & 300 & 17 & 5.67 \\
\hline East China & Jiangsu & Jiang et al. [41] & IHA & 1991 & 212 & 12 & 5.66 \\
\hline East China & Jiangsu & Chen et al. [42] & IHA & 1998 & 110 & 1 & 0.91 \\
\hline East China & Jiangsu & Wu et al. [43] & IHA & 1994 & 1129 & 17 & 1.51 \\
\hline East China & Jiangsu & Zhu et al. [44] & IHA & 1994 & 3542 & 156 & 4.40 \\
\hline East China & Jiangsu & Zhu et al. [45] & ELISA & 1997 & 800 & 21 & 2.63 \\
\hline East China & Jiangsu & Yuan et al. [46] & ELISA & 1998 & 723 & 15 & 2.07 \\
\hline East China & Jiangsu & Liu et al. [47] & ELISA & 2001 & 2589 & 78 & 3.01 \\
\hline East China & Anhui & Wang et al. [48] & ELISA & 1999 & 670 & 19 & 2.84 \\
\hline East China & Anhui & Shen et al. [49] & ELISA & 2000 & 638 & 39 & 6.11 \\
\hline East China & Zhejiang & Meng et al. [50] & ELISA & 1996 & 1197 & 215 & 17.96 \\
\hline East China & Zhejiang & Jiang et al. [51] & ELISA & 2006 & 1023 & 58 & 5.67 \\
\hline South China & Guangdong & Zeng et al. [52] & ELISA & 2005 & 680 & 49 & 7.21 \\
\hline South China & Guangdong & Zhong et al. [53] & ELISA & 2010 & 1000 & 94 & 9.40 \\
\hline South China & Guangdong & $\mathrm{Gu}$ et al. [54] & ELISA & 2010 & 4500 & 69 & 1.53 \\
\hline South China & Guangxi & Huang et al. [55] & ELISA & 2013 & 2510 & 74 & 2.95 \\
\hline
\end{tabular}

Concerning the meta-regression results for prevalence in different years, Figure 4 shows that there was no statistically significant difference in terms of the prevalence trends $(p>0.05)$. The lowest and highest prevalence was $1.07 \%$ (95\% CI: $0.55 \%-1.73 \%)$ in 1991 and $21.21 \%(95 \% \mathrm{CI}$ : $16.48 \%-26.36 \%$ ) in 2001 .

\section{Seroprevalence of $\boldsymbol{T}$. gondii infection in blood donors in relation to risk factors}

The pooled seroprevalence for each subgroup was calculated using the random-effects model, if there was high heterogeneity. The pooled estimates by potential various risk factors associated with $T$. gondii infection in blood donors are presented in Table 3. The seroprevalence of T. gondii tested with the ELISA method was $7.30 \%$ (95\% CI: $5.25 \%-9.67 \%$ ), and $3.16 \%(95 \%$ CI: $1.87 \%-4.76 \%)$ with the IHA method, and the difference between the two methods was significant $(p=0.002)$. The seroprevalence of $T$. gondii infection for different educational levels was significantly different ( $p=0.006$ ), with $4.80 \%$ (95\% CI: $3.44 \%-6.37 \%$ ) in the university-level population, 6.58\% (95\% CI: 4.79\%-8.63\%) in the high school population, and 9.01\% (95\% CI: $6.89 \%-$ $11.38 \%$ ) in the $\leq$ middle school population. There was no relationship between age, gender, occupation or blood type and seroprevalence of T. gondii $(p>0.05)$.

Given the obvious difference between the two screening methods, we performed the analysis separately. Among the nine studies using the IHA method, sex data was provided in only three studies. We compared seroprevalence using different methods in men and women separately. In men, the seroprevalence using the ELISA method was $7.53 \%(4.81 \% ; 10.78 \%)$, and using IHA $2.65 \%(0.52 \% ; 6.23 \%)$. In women, the seroprevalence using the ELISA method was $6.78 \%(4.38 \%$; $9.66 \%)$, and using IHA $2.11 \%(0.85 \% ; 3.84 \%)$. The difference between the sexes was significant $(p<0.05)$. 
Study

Ai et al. (2000)

Chen et al. (1990)

Chen et al. (1999)

Feng et al. (1998)

Gu et al. (1989)

Gu et al. (2010)

Hu et al. (1991)

Huang et al. (2012)

Jiang et al. (1990)

Jiang et al. (2006)

Kuang et al. (2002)

$\mathrm{Li}$ et al. (2003)

Liu et al. (2000)

Luo et al. (2003)

Meng et al. (1996)

Shen et al. (2000)

Shen et al. (2017)

Song et al. (2009)

Song et al. (2010)

Sun et al. (1990)

Sun et al. (2013)

Tong et al. (1991)

Wang et al. (1996)

Wang et al. (1999)

Wang et al. (2001)

Wang et al. (2014)

Wu et al. (1989)

Wu et al. (1993)

Wu et al. (2016)

Xin et al. (2013)

Xu et al. (2017)

Yang et al. (1995)

Yang et al. (2012)

Yuan et al. (1998)

Zeng et al. (2005)

Zhong et al. (2010)

Zhu et al. (1986)

Zhu et al. (1994)

Zhu et al. (1997)

Zhu et al. (2007)

Random effects model

Heterogeneity: $I^{2}=98 \%, \tau^{2}=0.0131, p<0.01$

Events Total

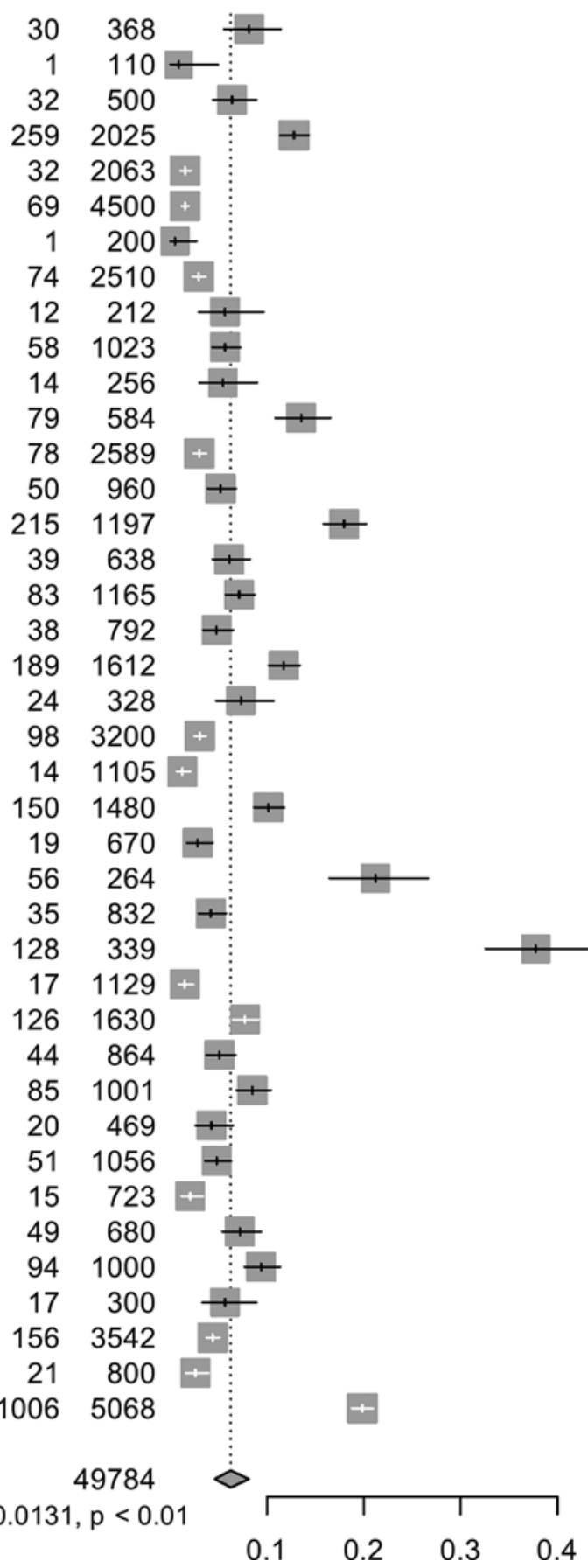

Proportion $95 \%-\mathrm{Cl}$ Weight

0.0815 [0.0557; 0.1143] $2.5 \%$

$0.0091[0.0002 ; 0.0496] \quad 2.2 \%$

$0.0640[0.0442 ; 0.0892] \quad 2.5 \%$

$0.1279[0.1137 ; 0.1432] \quad 2.6 \%$

$0.0155[0.0106 ; 0.0218] \quad 2.6 \%$

$0.0153[0.0119 ; 0.0194] \quad 2.6 \%$

$0.0050[0.0001 ; 0.0275] \quad 2.4 \%$

$0.0295[0.0232 ; 0.0369] \quad 2.6 \%$

$0.0566[0.0296 ; 0.0968] \quad 2.4 \%$

$0.0567[0.0433 ; 0.0727] \quad 2.5 \%$

$0.0547[0.0302 ; 0.0901] \quad 2.4 \%$

$0.1353[0.1086 ; 0.1657] \quad 2.5 \%$

$0.0301[0.0239 ; 0.0375] \quad 2.6 \%$

$0.0521[0.0389 ; 0.0681] \quad 2.5 \%$

$0.1796[0.1583 ; 0.2026] \quad 2.5 \%$

$0.0611[0.0438 ; 0.0826] \quad 2.5 \%$

$0.0712[0.0571 ; 0.0876] \quad 2.5 \%$

$0.0480[0.0342 ; 0.0653] \quad 2.5 \%$

$0.1172[0.1019 ; 0.1340] \quad 2.5 \%$

$0.0732[0.0474 ; 0.1069] \quad 2.4 \%$

$0.0306[0.0249 ; 0.0372] \quad 2.6 \%$

$0.0127[0.0069 ; 0.0212] \quad 2.5 \%$

$0.1014[0.0864 ; 0.1179] \quad 2.5 \%$

$0.0284[0.0172 ; 0.0439] \quad 2.5 \%$

$0.2121[0.1644 ; 0.2664] \quad 2.4 \%$

$0.0421[0.0295 ; 0.0580] \quad 2.5 \%$

$0.3776[0.3258 ; 0.4316] \quad 2.4 \%$

$0.0151[0.0088 ; 0.0240] \quad 2.5 \%$

$0.0773[0.0648 ; 0.0913] \quad 2.5 \%$

$0.0509[0.0372 ; 0.0678] \quad 2.5 \%$

$0.0849[0.0684 ; 0.1039] \quad 2.5 \%$

$0.0426[0.0262 ; 0.0651] \quad 2.5 \%$

$0.0483[0.0362 ; 0.0630] \quad 2.5 \%$

$0.0207[0.0117 ; 0.0340] \quad 2.5 \%$

$0.0721[0.0538 ; 0.0941] \quad 2.5 \%$

$0.0940[0.0766 ; 0.1138] \quad 2.5 \%$

$0.0567[0.0334 ; 0.0892] \quad 2.4 \%$

$0.0440[0.0375 ; 0.0513] \quad 2.6 \%$

$0.0262[0.0163 ; 0.0398] \quad 2.5 \%$

$0.1985[0.1876 ; 0.2098] \quad 2.6 \%$

$0.0626[0.0462 ; 0.0813] 100.0 \%$

Fig. 2. Forest plot of the overall seroprevalence estimates of $T$. gondii in blood donors.

\section{Publication bias and sensitivity tests}

Funnel plot and Egger's test were both used to examine publication bias. As shown in Figure 5, the funnel plot indicates no publication bias, which was also confirmed from Egger's test $(t=0.11, p=0.91)$. A sensitivity analysis was conducted for the pooled results by converting the pooled model (from the random effects model to the fixed effects model). The results demonstrated no large differences in proportions and $95 \%$ CIs before and after pooling, indicating stability in the pooled results.

\section{Discussion}

Although T. gondii infection in China has been studied for 60 years [24] and many papers have investigated the prevalence of T. gondii in different populations, including blood 


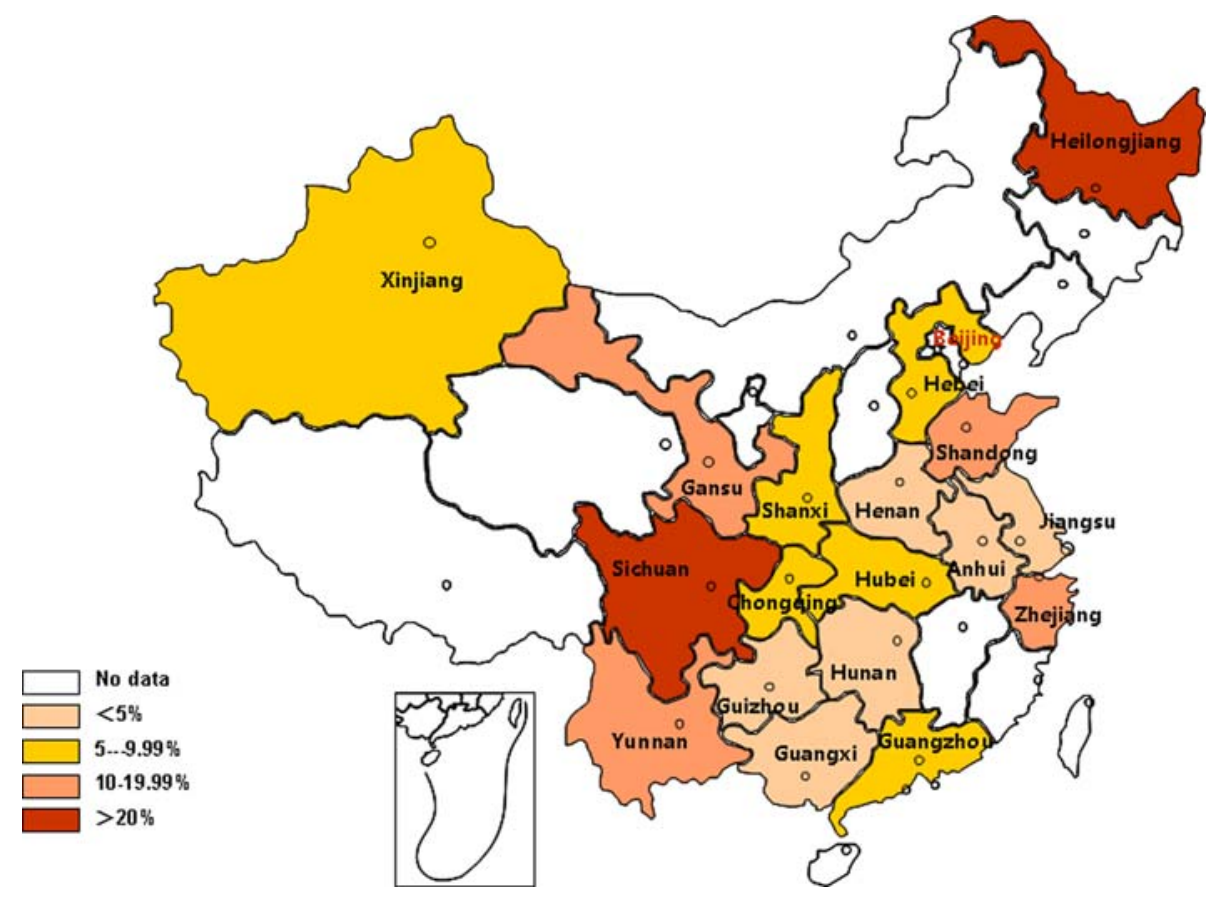

Fig. 3. Geographic distribution of $T$. gondii prevalence.

Table 2. Comparison of prevalence rates in different regions

\begin{tabular}{|c|c|c|c|c|c|}
\hline \multirow[b]{2}{*}{ Regions } & \multirow[b]{2}{*}{ No. of studies } & \multirow[b]{2}{*}{ No. of donors } & \multirow[b]{2}{*}{ Prevalence $[95 \% \mathrm{CI}](\%)$} & \multicolumn{2}{|c|}{ Heterogeneity test } \\
\hline & & & & $I^{2}(\%)$ & $p$-value \\
\hline Northwest & 3 & 2176 & $8.95[7.29 ; 10.76]$ & 37.40 & 0.20 \\
\hline East China & 13 & 14,958 & $4.85[2.78 ; 7.43]$ & 97.60 & $<0.01$ \\
\hline Southwest & 5 & 7108 & $11.93[4.54 ; 22.16]$ & 98.70 & $<0.01$ \\
\hline Central China & 7 & 8637 & $4.24[2.25 ; 6.82]$ & 96.00 & $<0.01$ \\
\hline South China & 4 & 8690 & $4.71[1.88 ; 8.71]$ & 98.00 & $<0.01$ \\
\hline North China & 7 & 7951 & $6.36[4.55 ; 8.45]$ & 92.20 & $<0.01$ \\
\hline Northeast & 1 & 264 & $21.21[16.48 ; 26.36]$ & & \\
\hline Total & 40 & 49,784 & $6.26[4.62 ; 8.13]$ & 98.00 & $<0.01$ \\
\hline
\end{tabular}

donors, no systematic review on $T$. gondii in blood donors was carried out. In this study, we searched databases and identified a total of 40 relevant articles which contained eligible data on the seroprevalence of $T$. gondii infection in 49,784 blood donors across mainland China. To our knowledge, this is the first report to evaluate the national level of $T$. gondii seroprevalence in blood donors, which could be of great importance to public health surveillance and associated control policies.

The overall seroprevalence of $T$. gondii infection in blood donors in mainland China from 1986 to 2017 was $6.26 \%$ (95\% CI: $4.62 \%-8.13 \%$ ). Our study showed a low seroprevalence of $T$. gondii infection in blood donors in mainland China compared to the average seroprevalence of $33 \%$ (95\% CI: $28 \%-39 \%$ ) worldwide [8]. Compared to the prevalence recorded in other Asia countries, the prevalence of $T$. gondii in China was the lowest [8].

T. gondii is widely distributed, especially in warm, moist and low altitude regions, and at temperate to tropical temperatures oocysts remain infectious for up to 1.5 years [20]. In this study, it was interesting to note that the cold northeast regions of China, at high attitude, had the highest prevalence, and the warm and low attitude regions in the south of China had the lowest prevalence. The result was consistent with Pan's review [24]. This may be related to economic development levels and sanitary conditions. Another reason was that the investigations available for the north of China were few; in some regions, only one province reported the prevalence of $T$. gondii infection.

In our research, there were two factors, namely screening methods and education levels, which were associated with T. gondii seroprevalence. An experiment that compared IHA, ELISA and another screening method with each other found that there was no significant difference in sensitivity and specificity between IHA and ELISA [50]. However, we found that testing methods may be one of the main sources of heterogeneity in this meta-analysis. In addition, it may be caused by the sample size and/or other confounding factors. Worldwide, T. gondii infection is associated with gender, age, contact with animals and raw meat consumption [8], but not with blood group. However, in Iran, the difference between men and 


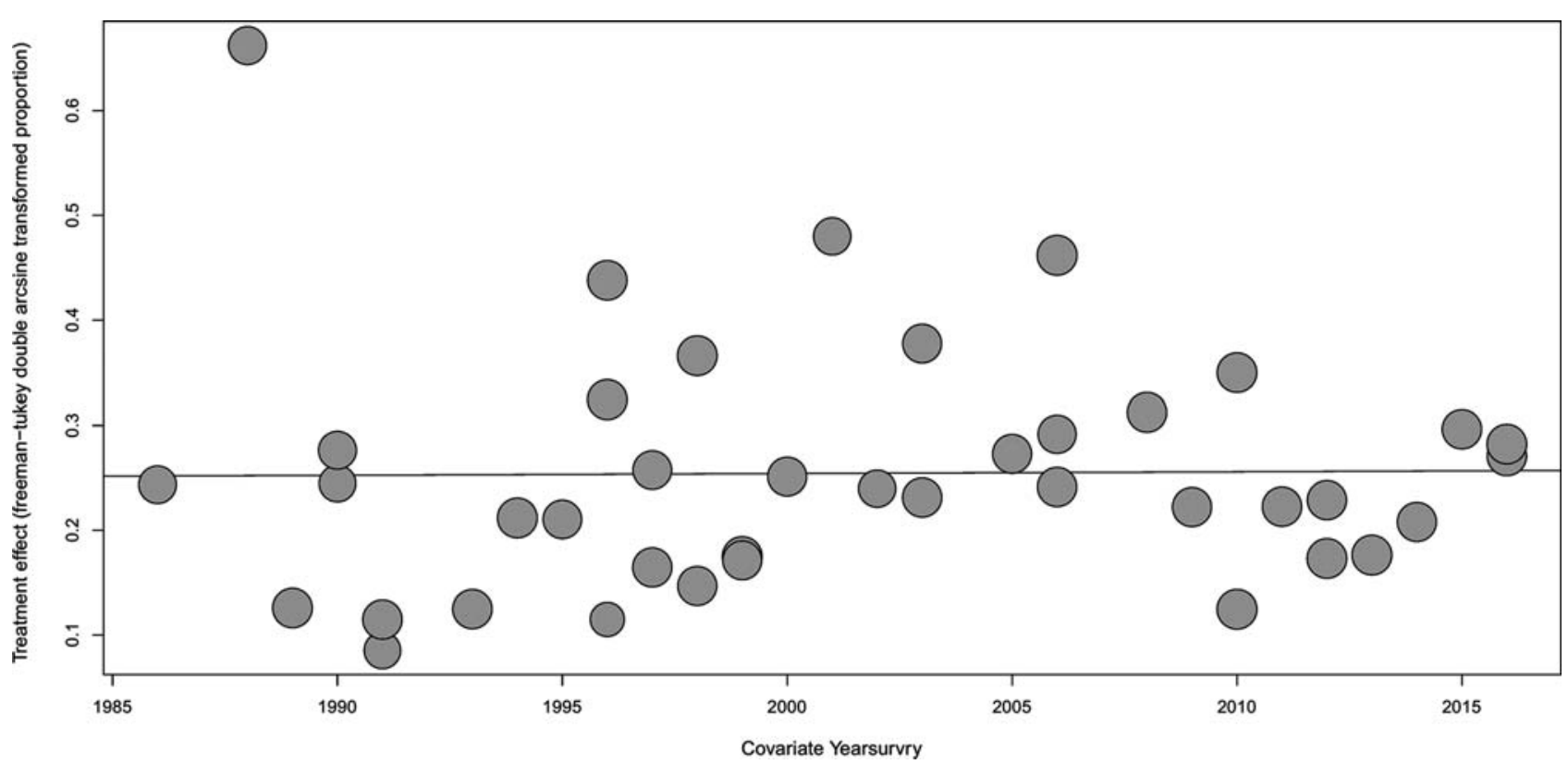

Fig. 4. Meta-regression plot of antibodies to T. gondii according to the year of study. The overall prevalence of antibodies against $T$. gondii increased according to the year of study, but the trend was not significant $(p>0.05)$.

Table 3. Seroprevalence of $T$. gondii in blood donors associated with risk factors.

\begin{tabular}{|c|c|c|c|c|c|c|c|c|c|}
\hline \multirow[b]{2}{*}{ Factors } & \multirow[b]{2}{*}{ Categories } & \multirow{2}{*}{$\begin{array}{l}\text { No. of } \\
\text { studies }\end{array}$} & \multirow{2}{*}{$\begin{array}{c}\text { No. of blood } \\
\text { donors }\end{array}$} & \multirow[b]{2}{*}{ No. of $\operatorname{IgG}(+)$} & \multirow{2}{*}{$\begin{array}{c}\text { Prevalence } \\
{[95 \% \text { CI] }(\%)}\end{array}$} & \multicolumn{2}{|c|}{ Heterogeneity } & \multicolumn{2}{|c|}{$\begin{array}{l}\text { Between-group } \\
\text { differences* }\end{array}$} \\
\hline & & & & & & $I^{2}$ & $p$-value & $Q$ & $p$-value \\
\hline \multirow{3}{*}{ Method } & & & & & & & & 9.46 & 0.0021 \\
\hline & ELISA & 31 & 41431 & 3298 & $7.30[5.25 ; 9.67]$ & $98.70 \%$ & $<0.01$ & & \\
\hline & IHA & 9 & 8353 & 280 & $3.16[1.87 ; 4.76]$ & $90.40 \%$ & $<0.01$ & & \\
\hline \multirow[t]{4}{*}{ Age } & & & & & & & & 0.99 & 0.6082 \\
\hline & $18-30$ & 15 & 15582 & 1471 & $7.37[4.02 ; 11.61]$ & $98.70 \%$ & $<0.01$ & & \\
\hline & $30-40$ & 15 & 5458 & 469 & $8.49[5.87 ; 11.53]$ & $92.60 \%$ & $<0.01$ & & \\
\hline & $>40$ & 15 & 2903 & 270 & $9.36[6.10 ; 13.17]$ & $88.70 \%$ & $<0.01$ & & \\
\hline \multirow[t]{3}{*}{ Gender } & & & & & & & & 0.07 & 0.7983 \\
\hline & Male & 22 & 16652 & 1369 & $6.75[4.31 ; 9.67]$ & $97.90 \%$ & $<0.01$ & & \\
\hline & Female & 22 & 14545 & 1104 & $6.21[4.01 ; 8.83]$ & $97.10 \%$ & $<0.01$ & & \\
\hline \multirow[t]{5}{*}{ Occupation } & & & & & & & & 1.84 & 0.6061 \\
\hline & Students & 10 & 5152 & 332 & $4.47[1.83 ; 8.13]$ & $96.30 \%$ & $<0.01$ & & \\
\hline & Job-holders & 10 & 6355 & 536 & $5.99[2.57 ; 10.64]$ & $97.50 \%$ & $<0.01$ & & \\
\hline & Farmers & 10 & 4611 & 561 & $8.49[3.91 ; 14.54]$ & $97.50 \%$ & $<0.01$ & & \\
\hline & Others & 8 & 2356 & 188 & $6.46[3.22 ; 10.65]$ & $92.00 \%$ & $<0.01$ & & \\
\hline \multirow[t]{5}{*}{ Blood } & & & & & & & & 0.01 & 0.9997 \\
\hline & A & 3 & 1421 & 104 & $7.74[2.38 ; 15.69]$ & $94.60 \%$ & $<0.01$ & & \\
\hline & B & 3 & 1773 & 140 & $7.36[1.84 ; 15.99]$ & $96.40 \%$ & $<0.01$ & & \\
\hline & $\mathrm{AB}$ & 3 & 406 & 28 & $6.97[0.05 ; 21.35]$ & $92.80 \%$ & $<0.01$ & & \\
\hline & $\mathrm{O}$ & 3 & 1382 & 95 & $7.23[1.51 ; 16.50]$ & $95.80 \%$ & $<0.01$ & & \\
\hline \multirow[t]{4}{*}{ Education } & & & & & & & & 10.29 & 0.0058 \\
\hline & University & 3 & 849 & 41 & $4.80[3.44 ; 6.37]$ & $0.00 \%$ & 0.63 & & \\
\hline & High school & 3 & 2361 & 161 & $6.58[4.79 ; 8.63]$ & $72.00 \%$ & 0.03 & & \\
\hline & $\leq$ Middle school & 3 & 641 & 58 & $9.01[6.89 ; 11.38]$ & $0.00 \%$ & 0.72 & & \\
\hline
\end{tabular}

${ }^{*}$ Test for subgroup differences using random effects model.

women was not statistically significant [19]. In our study, lower educational level blood donors had higher seroprevalence, which may be related to the living conditions and chances of contact with animals.
There remain some limitations in our study. First, the articles in this current study were mostly published ten to twenty years ago; only six papers were published in the last five years. This shows that less attention has been focused on $T$. gondii 

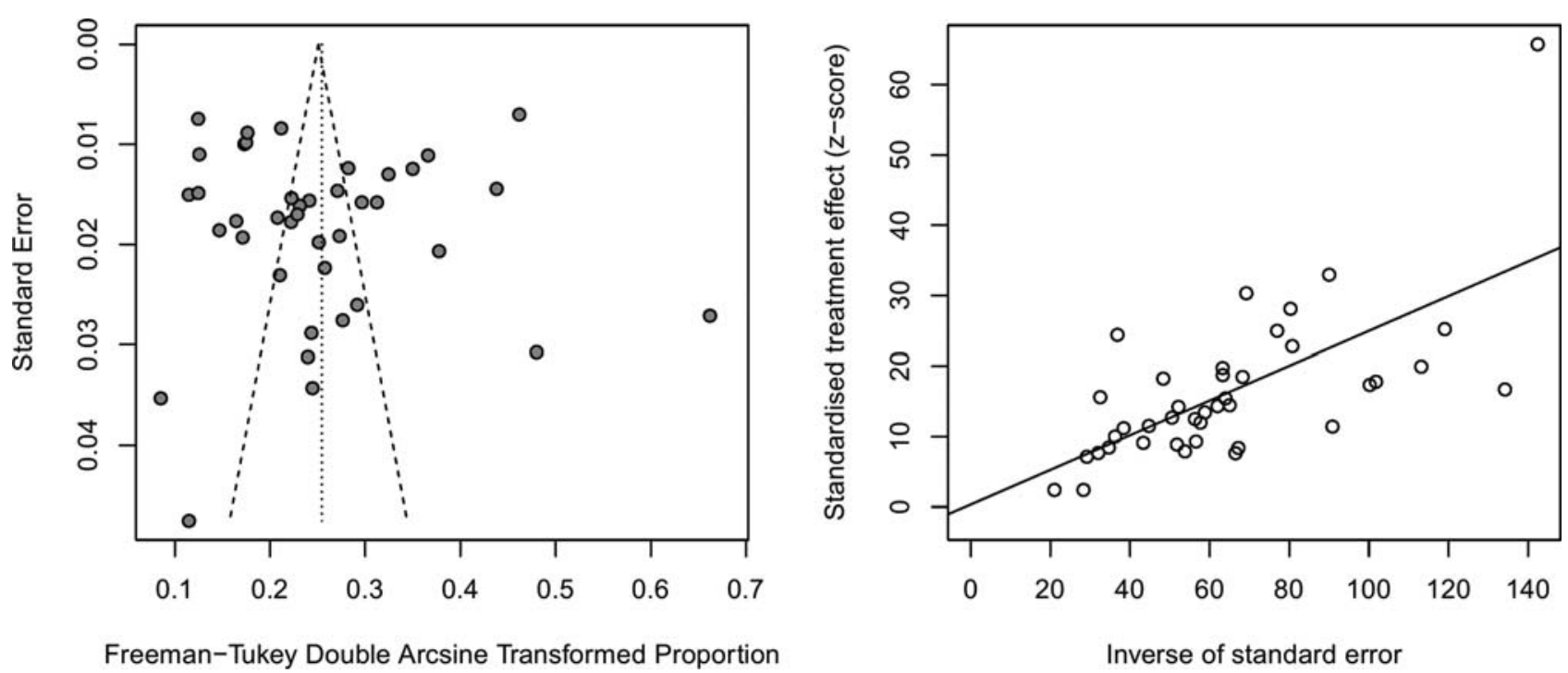

Fig. 5. Funnel plot (left) and Egger's publication bias plot (right), showing that no potential publication bias existed.

infection in blood donors in recent years. Second, most of the studies focused on seroprevalence and the methods were varied; only two of them selectively detected DNA positivity for T. gondii [7, 59]. In addition, there was no investigation of the patients who received DNA-positive blood. Therefore, it could not be determined whether there was a possibility of transfusion-transmitted toxoplasmosis. Third, in terms of risk factors, only the characteristics of blood donors were analyzed in most of studies Risk factors like contact with animals (cats or dogs) or eating uncooked or raw meat and vegetables were not investigated in the articles. Fourth, to make the data comparable and to minimize the heterogeneity, we only adopted $\mathrm{IgG}$ as an indicator to analyze the prevalence of $T$. gondii infection. In diagnosis of $T$. gondii infection, the most useful indicator of active infection may be $\operatorname{IgG}$ and/or IgM. In this analysis of 40 studies, IgG and/or IgM was used in only 19 studies, IgM was used in two additional studies [5, 46]; the data about IgG and $\operatorname{IgM}$ need to be explored further.

In conclusion, the prevalence of antibodies to $T$. gondii in Chinese blood donors was lower than in other countries. However, the risk of transfusion-transmitted toxoplasmosis still exits. Only in one report, two patients were confirmed to have transfusion-transmitted toxoplasmosis from May 1986 to 1989 in China [36]. Although direct identification methods of T. gondii infection have been developed, routine use is not available in blood banks. In most Chinese blood centers, blood is processed by leukocyte filtration, which reduces the number of mandatory intracellular pathogens including $T$. gondii. The risk of $T$. gondii infection was also greatly reduced. Based on this, it is not necessary to screen $T$. gondii in blood donations in China.

\section{Conflict of interest}

The authors declare that they have no conflict of interests.

Acknowledgements. The authors would like to thank Jiangsu Province Science and Technology Department (No. 2016-III-3345), Jiangsu Province Commission of Health and Family Planning
(No. LGY2016045), and the National Natural Science Foundation of China (No. 81402433) for their support of this study.

\section{References}

1. Ai C, Hui Q, Sun X, Xue Y, Jing C. 2007. Investigation and research on Toxoplasma gondii infection of voluntary blood donors in Yan'an City. Shanxi Medical Journal, 36, 884-886.

2. Barendregt JJ, Doi SA, Lee YY, Norman RE, Vos T. 2013. Meta-analysis of prevalence. Journal of Epidemiology Community Health, 67(11), 974-978.

3. Chen Q, Liu N. 1991. Investigation and clinic significance of Toxoplasma gondii by IHA in Qidong. Chinese Journal of Zoonoses, 7, 37.

4. Chen Y, Lang S, Li J, Qiu X. 1999. An epidemiological survey of opportunity pathogenic parasites in Guiyang. Journal of Guiyang Medical College, 24, 243-245.

5. Chen Z, Wang C, Du Q, Ni L, Xie H. 1998. Serological investigation of Toxoplasma gondii and anti cytomegalovirus IgM antibodies in blood donors in Nanjing. Chinese Journal of Zoonoses, 14(4), 2.

6. Egger M, Davey Smith G, Schneider M, Minder C. 1997. Bias in meta-analysis detected by a simple, graphical test. British Medical Journal, 315(7109), 629-634.

7. Feng G, Du H, Mao Y, Zhen J, Yu J, Zhen C, Wu W, Huang Y, Li G. 1998. Investigation of CMV and Tox antibody target to 2025 donors. Shanxi Journal of Medical Laboratory Sciences, 13, 3-5.

8. Foroutan-Rad M, Majidiani H, Dalvand S, Daryani A, Kooti W, Saki J, Hedayati-Rad F, Ahmadpour E. 2016. Toxoplasmosis in blood donors: A systematic review and meta-analysis. Transfusion Medicine Reviews, 30(3), 116-122.

9. Gu R, Chen L, Chen H, Liu K, Zhou J, Yang S. 1989. Prevalence of Toxoplama gondii in blood donors in some regions of Hubei. Chinese Journal of Blood Transfusion, 2, 194-195.

10. Gu X, Zeng J, Wang L. 2010. Diagnosis of Toxoplasma gondii Infection with ELISA. Practical Preventive Medicine, 17(9), 3.

11. Hu L, Shen J. 1991. Clinical investigation on seroprevalence of Toxoplasmosis. Chinese Journal of Zoonoses, 7(5), 38. 
12. Huang Z, Yang S, Liang R. 2013. Investigation on Toxoplasma gondii infection in blood donors in 2012. Henan Traditional Chinese Medicine, 10, 245-246.

13. Jiang G, Wu G, Sang L, Ru X. 2006. Serological investigation on Toxoplasma gondii infection in Shaoxing blood donors. Chinese Journal of Health Laboratory Technology, 16, 859.

14. Jiang Q, Jiang X, Ma F, Ge Q. 1991. Investigation on Toxoplasma gondii infection in blood donors in Danyang, Jiangsu. Chinese Journal of Zoonoses, 7, 37.

15. Kuang ES, Yang FH, Xiang-Wan HE, Liu J, Yan YF. 2002. Experimental studies and comparison on clinic diagnosis of ToRCH IgM antibody. Virologica Sinica, 17(1), 56-60.

16. Li Y, Fu W, Yu Z, Cheng G. 2003. Investigation and analysis on Toxoplasma gondii antibodies in 584 blood donors. Chinese Journal of Blood Transfusion, 16, 278-279.

17. Liu Y, Zheng K, Chen M, Fu L, Du W, Shi Z. 2001. Investigation on Toxoplasma gondii infection in blood donors in Xuzhou. Acta Academiae Medicine Xuzhou, 21, 45-47.

18. Luo X, Guo D. 2003. Serological investigation of Toxoplasma gondii infection in university students. Chinese Journal of Epidemiology, 24, 71.

19. Mansouri A, Adhami Mojarad MR, Badfar G, Abasian L, Rahmati S, Kooti W, YektaKooshali MH, Soleymani A, Azami M. 2017. Epidemiology of Toxoplasma gondii among blood donors in Iran: A systematic review and meta-analysis. Transfusion and Apheresis Science, 56(3), 404-409.

20. Martin P, Bouza P. 2004. Blood and tissue protozoa, in Infectious diseases, 2nd edn. Cohen J, Powderly WG, Editors. Mosby: Philadelphia, PA.

21. Meng Z, Ye X, Zhang L, Lin A. 1996. Serological investigation on Toxoplasma gondii infection in 1197 blood donors in Zhejiang Province. Chinese Journal of Blood Transfusion, 9, 43-44.

22. Ministry ohotPsRoC. Blood Donor Physical Examination Requirements (GB18467-2001).

23. Moher D, Liberati A, Tetzlaff J, Altman DG, Group P. 2009. Preferred reporting items for systematic reviews and meta-analyses: the PRISMA statement. PLOS Medicine, 6(7), e1000097.

24. Pan M, Lu C, Zhao J, Shen B. 2017. Sixty years (1957-2017) of research on toxoplasmosis in china - An overview. Frontiers in Microbiology, 8, 1825.

25. Robert-Gangneux F, Dardé ML. 2012. Epidemiology of and diagnostic strategies for toxoplasmosis. Clinical Microbiology Reviews, 25(2), 264-296.

26. Schwarzer G. 2007. Meta: An R package for meta-analysis. R News, 7(3), 40-44.

27. Shen Y, Wang H. 2000. Investigation on Toxoplasma gondii infection in 638 blood donors. Labeled Immunoassays and Clinical Medicine, 3, 179.

28. Shen Y, Yang Y, Chen Y, Song X. 2017. Survey of prevalence of Toxoplasma gondii among the healthy unpaid blood donation population in Baixiang of Hebei Province. Journal of Medical Pest Control, 33(10), 2.

29. Siegel SE, Lunde MN, Gelderman AH, Halterman RH, Brown JA, Levine AS, Graw RG Jr. 1971. Transmission of toxoplasmosis by leukocyte transfusion. Blood, 37(4), 388-394.

30. Song R. 2009. Analysis on Toxoplasma gondii in different blood donors populations. Chinese Journal of Blood Transfusion, 22, 835-836.

31. Song R. 2012. Relationship between toxoplasma IgG and routine screening items of infectious diseases of blood donors. Chinese Journal of Schistosomiasis Control, 24(3), 371-372.
32. Sun A, Wang W. 2015. Analysis on the test results of Toxoplasma gondii antibody in volunteer blood donors. Chinese Journal of Blood Transfusion, 370(1661), 20140033.

33. Sun X, Sun A, Zhang Y. 1991. Antibodies of Toxoplasma gondii detection in blood donors in Yining. Xiangjiang Laboratory Medicine, 6, 129.

34. Tenter AM, Heckeroth AR, Weiss LM. 2000. Toxoplasma gondii: from animals to humans. International Journal of Parasitology, 30(12-13), 1217-1258.

35. Tong $\mathrm{H}, \mathrm{Ou}$ Y. 1994. Investigation of Toxoplasma gondii infection in Hunan blood donors. Chinese Journal of Blood Transfusion, 7, 40.

36. Wang C, Huang C, Wang J, Jin Z, Zhu Y, Shen J, Hang P, Zhang D, Tang X, Zhu Q, Gong P, Wang X. 1994. Transfusion and Toxoplasma gondii infection. Chinese Journal of Zoonoses, 10(4), 45-46.

37. Wang $\mathrm{H}, \mathrm{Xu}$ J. 1999. Investigation on Toxoplasma gondii infection in 670 blood donors. Journal of Clinical Transfusion and Laboratory Medicine, 1, 40.

38. Wang Y, Li X. 1998. Investigation of Toxoplasma gondii infection in blood donors in Lanzhou. Medical Journal of National Defending Forces in Northwest China, 19, 124.

39. Wang Y, Liu X, Ren L, Yang F. 2002. Investigation on Toxoplasma gondii infection in 264 blood donors. Journal of Mudanjiang Medical College, 23, 37.

40. Wang YR, Shi HL, Chen YK, Yang YX, Song RH. 2014. Serological survey of Toxoplasma infection in healthy blood donation population. Medical Recapitulate, 20(21), 3992-3993.

41. Wu J. 1994. Investigation on Toxoplasma gondii infection in 1129 blood donors. Chinese Journal of Schistosomiasis Control, 6(6), 350.

42. Wu Y, Song R. 2017. Survey of Toxoplasma gondii infection characteristics and its risk factors among healthy blood donation population in Shijiazhuang City, Hebei Province. Chinese Journal of Schistosomiasis Control, 29, 377-379.

43. Xiao Y, Yin J, Jiang N, Xiang M, Hao L, Lu H, Sang H, Liu X, Xu H, Ankarklev J, Lindh J, Chen Q. 2010. Seroepidemiology of human Toxoplasma gondii infection in China. BMC Infectious Diseases, 10, 4.

44. Xie T. 1964. A case report of human toxoplasmosis. Jiangxi Medicine, 4, 121-122.

45. Xin LF, Song RH. 2013. Prevalence of IgG antibodies specific to Toxoplasma gondii among college student blood donors in Shijiazhuang City. Chinese Journal of Schistosomiasis Control, 25(3), 254, 258.

46. Xu L, Sheng X. 2000. Serological investigation and analysis of circulating Toxoplasma gondii (CAg) in 1213 blood donors. Modern Preventive Medicine, 27(3), 1.

47. Xu M, Mao W, He T, Zhang C, Wei Y, Gao Z, Zhang Y, He M. 2017. Investigation and research on Toxoplasma gondii infection of volunteer blood donors in Chongqing. China. Chinese Journal of Blood Transfusion, 30(2), 3.

48. Yang L, Shi C, Feng Z, Chen H, Wang X. 1995. Investigation on Toxoplasma gondii antibodies in 469 blood donors. Chinese Journal of Blood Transfusion, 8, 152-153.

49. Yang SJ, Song RH. 2012. Seroprevalence of Toxoplasma gondii antibodies in healthy voluntary blood donors from Shijiazhuang area. Chinese Journal of Schistosomiasis Control, 24(6), 728, 730.

50. Yang YX, Chen YK, Wei SJ, Song RH. 2014. Efficiency of three methods for detecting Toxoplasma IgG antibody. Chinese Journal of Schistosomiasis Control, 26(1), 109-110. 
51. Yu E, Chen T, Lin S. 1957. The occurrence of Toxoplasma in cats and rabbits in Fukien. China Acta Microbiologica Sinica, 5, 101-102.

52. Yu S, Xu L, Jiang Z, Xu S, Han J, Zhu Y, Chang J, Lin J, Funiu X. 1994. Report on the first national wide survey of the distribution of human parasites in China. Chinese Journal of Parasitology and Parasitic Diseases, 12, 241-247.

53. Yuan C, He H, Xu F, Su C, Zhuang H. 1998. Investigation on Toxoplasma gondii infection in 723 blood donors in Lianyungang. Journal of Radioimmunology, 11, 386-387.

54. Zeng JF, Cai H. 2005. Seroepidemiological survey of Toxoplasma gondii in infection in blood donors in Shenzhen City. China Tropical Medicine, 5(3), 3.

55. Zhong CC, Shi DM, Lai FC, Kuang JP. 2010. Study on Toxoplasma gondii infection in Zhuhai area blood donors. International Medicine \& Health Guidance News, 16(4), 4.

56. Zhou P, Chen N, Zhang RL, Lin RQ, Zhu XQ. 2008. Foodborne parasitic zoonoses in China: perspective for control. Trends in Parasitology, 24, 190-196.
57. Zhou P, Chen Z, Li HL, Zheng H, He S, Lin RQ, Zhu XQ. 2011. Toxoplasma gondii infection in humans in China. Parasites \& Vectors, 4, 165.

58. Zhu XM, Yang TH, Yang GQ. 2007. Study on Toxoplasma gondii infection in blood donors of Yunnan Province. Journal of Clinical Transfusion \& Laboratory Medicine, 9(4), 4.

59. Zhu Y, Hang P, Zhu Q, Wang J, Wang C. 1987. Preliminary investigation of Toxoplasma gondii infection. Chinese Journal of Public Health, 3, 81.

60. Zhu Y, Hang P, Zhu Q, Wang J, Wang C. 1989. Investigation on Toxoplasma gondii infection in 339 blood donors. Chinese Journal of Transfusion, 2, 192-194.

61. Zhu Y, Wang J, Cen W, Zhou Y, Wang C. 1994. Investigation on Toxoplasma gondii infection in blood donors in Jiangsu. Chinese Journal of Schistosomiasis Control, 6, 108-109.

62. Zhu Z, Yin Y, Chen L, Shen M, Zhang H. 1997. Investigation on Toxoplasma gondii infection in blood donors in Kunshan. Journal of Radioimmunology, 9, 1003.

Cite this article as: Wang T, Han Y, Pan Z, Wang H, Yuan M \& Lin H: Seroprevalence of Toxoplasma gondii infection in blood donors in mainland China: a systematic review and meta-analysis. Parasite, 2018, 25, 36.

\section{O PARASTE}

An international open-access, peer-reviewed, online journal publishing high quality papers on all aspects of human and animal parasitology

Reviews, articles and short notes may be submitted. Fields include, but are not limited to: general, medical and veterinary parasitology; morphology, including ultrastructure; parasite systematics, including entomology, acarology, helminthology and protistology, and molecular analyses; molecular biology and biochemistry; immunology of parasitic diseases; host-parasite relationships; ecology and life history of parasites; epidemiology; therapeutics; new diagnostic tools.

All papers in Parasite are published in English. Manuscripts should have a broad interest and must not have been published or submitted elsewhere. No limit is imposed on the length of manuscripts.

Parasite (open-access) continues Parasite (print and online editions, 1994-2012) and Annales de Parasitologie Humaine et Comparée (1923-1993) and is the official journal of the Société Française de Parasitologie. 